\title{
A class of differential inverse quasi-variational inequalities in finite dimensional spaces
}

\author{
Wei Li, ${ }^{\mathrm{a}, \mathrm{b}}$, Yi-Bin Xiao ${ }^{\mathrm{c}, *}$, Nan-Jing Huang ${ }^{\mathrm{d}}$, Yeol Je Cho, ${ }^{\mathrm{e}, \mathrm{f}, *}$ \\ ${ }^{a}$ Geomathematics Key Laboratory of Sichuan Province, Chengdu University of Technology, Chengdu, Sichuan, 610059, P. R. China. \\ ${ }^{b}$ State Key Laboratory of Geohazard Prevention and Geoenvironment Protection, Chengdu, Sichuan, 610059, P. R. China. \\ ' School of Mathematical Sciences, University of Electronic Science and Technology of China, Chengdu, Sichuan, 611731, P. R. China. \\ ${ }^{d}$ Department of Mathematics, Sichuan University, Chengdu, 610064, P. R. China. \\ ${ }^{e}$ Department of Mathematics Education and the RINS, Gyeongsang National University, Jinju 660-701, Korea. \\ ${ }^{f}$ Center for General Education, China Medical University, Taichung 40402, Taiwan.
}

Communicated by Y. H. Yao

\begin{abstract}
In this paper, we introduce and study a class of differential inverse quasi-variational inequalities in finite dimensional Euclidean spaces, which are closely related to the differential variational inequalities. By using two important theorems on differential inclusions, we first prove some existence theorems for Carathéodory weak solutions of the differential inverse quasivariational inequality considered. Then, with the Euler computation method, we construct an Euler time-dependent scheme for solving the differential inverse quasi-variational inequality and prove a convergence result on the Euler time-dependent scheme constructed. (C)2017 All rights reserved.
\end{abstract}

Keywords: Differential inverse quasi-variational inequality, Carathéodory weak solution, Euler time-stepping scheme. 2010 MSC: 49J40, 35D30, 40A05.

\section{Introduction}

It is well-known that the inverse variational inequality introduced firstly by He and Liu in 2006 (see [10]) is closely related with the classic variational inequality which is studied widely by many researchers due to its important and wide applications in optimization, engineering, mechanics, economics, transportation, etc. (see for example [7, 23, 26-29, 32, 33]). The variables and the mappings in the inverse variational inequality are in the opposite positions of the classical variational inequality. As He and Liu pointed in [10], although the inverse variational inequality can be transformed into the classical variational inequality under some conditions, there are many control problems appearing in economics, transportation, management science, and energy networks that can be only modeled as inverse variational

\footnotetext{
*Corresponding author

Email addresses: lovelylw@126.com (Wei Li), xiaoyb9999@hotmail .com (Yi-bin Xiao), nanjinghuang@hotmail.com (Nan-jing Huang), yjchomath@gmail. com (Yeol Je Cho)

doi:10.22436/jnsa.010.08.45
} 
inequalities rather than classical variational inequalities. Therefore, many researchers devoted themselves to study various kinds of inverse variational inequalities and their generalizations. After introducing the inverse variational inequality, He et al. [9] studied a class of 'black-box' inverse variational inequalities and proposed some proximal point based algorithms to solve the class of inverse variational inequalities. In 2008, Yang [31] discussed the dynamic power price problem, in both the discrete and the evolutionary cases, and characterized the optimal price as a solution of an inverse variational inequality. Recently, Han et al. [8] proved two new existence theorems of solutions to the inverse variational and quasi-variational inequality problems considered using the KKM theorem and the Kakutani-Fan-Glicksberg fixed point theorem. We refer to $[11-13,22,30]$ and the reference therein for more research results on the inverse variational inequalities and their applications.

As an important and natural generalization of the inverse variational inequality, the inverse quasivariational inequalities is to find $u^{*} \in R^{n}$ such that $g\left(u^{*}\right) \in K\left(u^{*}\right)$ and

$$
\left\langle h\left(u^{*}\right), y-g\left(u^{*}\right)\right\rangle \geqslant 0, \quad \forall y \in K\left(u^{*}\right),
$$

where $h, g: R^{n} \rightarrow R^{n}$ are continuous mappings, $K: R^{n} \rightarrow 2^{R^{n}}$ is a set-valued mapping such that $K(u)$ is a closed convex subset in $R^{n}$ for each $u \in R^{n}$ and $\langle\cdot, \cdot\rangle$ denotes the inner product in $R^{n}$. In addition to the inverse quasi-variational inequalities, more and more generalizations of the inverse variational inequalities are studied in recent years. In 2013, Aussel [2] studied local and global error bounds for inverse quasi-variational inequality problems in terms of different gap functions and merit functions. $\mathrm{Li}$ and Zou [17] studied the existence of solution and error bounds for a class of inverse mixed quasivariational inequalities. For more research on the generalizations of inverse variational inequalities, we refer readers to $[8,15]$. For ease of writing in the rest of the paper, we denote the inverse quasi-variational inequality (1.1) and its solution set by IQVI $(h, g, K)$ and SIQVI $(h(\cdot), g(\cdot), K(\cdot))$, respectively.

The differential variational inequality, which is introduced by Pang and Steward in 2008 (see [20]), consists of two main components, i.e., an ordinary differential equation and a parametric variational inequality. The differential variational inequality unifies several mathematical problems such as ordinary differential equations, dynamic complementarity systems, differential algebraic equations and evolutionary variational inequalities. The differential variational inequality has many crucial applications in engineering and economics and thus are studied extensively in recent years (see for example [4$6,14,18,19,21,24,25])$. Naturally, the differential inverse variational inequality consists of an ordinary differential equation and a parametric inverse variational inequality, which has close relation with the differential variational inequality due to the relationship between the variational inequality and the inverse variational inequality. However, few researchers pay their attentions to the research on the differential inverse variational inequality and its generalizations. And thus there are few results available on the differential inverse variational inequality. Very recently, Li et al. [16] studied a class of differential inverse variational inequality in finite dimensional Euclidean spaces. An existence theorems of Carathéodory weak solutions and the linear growth of the solution set for the differential inverse variational inequality are proved. Also, an application of the differential inverse variational inequality to a time-dependent spatial price equilibrium control problem is given at last.

In this paper, we focus on the following generalization of the differential inverse variational inequality, which is called the differential inverse quasi-variational inequality (DIQVI):

$$
\left\{\begin{array}{l}
\dot{x}(t)=f(t, x(t))+B(t, x(t)) u(t), \\
u(t) \in \operatorname{SIQVI}(h(\cdot), G(t, x(t))+F(\cdot), K(\cdot)), \quad \forall t \in[0, T], \\
x(0)=x_{0},
\end{array}\right.
$$

where $x(t), u(t)$ are two functions on $[0, T], \dot{x}(t)=\frac{d x}{d t}$ stands for the derivative of function $x$ with respect to time variable $t, K: R^{n} \rightarrow 2^{R^{n}}$ is a set-valued mapping, $F: R^{n} \rightarrow R^{n}$ is a function, and $(f, B, G): \Omega \rightarrow$ $R^{m} \times R^{m \times n} \times R^{n}$ are given functions with $\Omega=[0, T] \times R^{n}$.

The remainder of this paper is organized as follows. In Section 2, we give some basic notations and preliminary results. Section 3 presents several existence theorems for weak solutions of DIQVI (1.2) in 
the sense of Carathéodory by applying two important results. At last, in Section 4, we construct an Euler time-dependent scheme for solving the DIQVI (1.2) and prove a convergence result on the Euler time-dependent scheme.

\section{Preliminaries}

In this section, we introduce some basic notations and preliminary results, which are useful in Sections 3 and 4 .

Definition 2.1 ([20]). A mapping $f: R^{n} \rightarrow R^{n}$ is said to be:

(1) monotone if

$$
\langle f(v)-f(u), v-u\rangle \geqslant 0, \quad \forall v, u \in R^{n} .
$$

(2) strongly monotone with a constant $\alpha>0$ if

$$
\langle\mathrm{f}(v)-\mathrm{f}(\mathrm{u}), v-\mathrm{u}\rangle \geqslant \alpha\|v-\mathrm{u}\|^{2}, \quad \forall v, \mathrm{u} \in \mathrm{R}^{\mathrm{n}} .
$$

Definition 2.2 ([2]). Let $g, f: R^{n} \rightarrow R^{n}$ be two mappings. $(f, g)$ is said to be a $\mu$-strongly monotone couple on $R^{n}$ if there exists a constant $\mu>0$ such that

$$
\langle f(x)-f(y), g(x)-g(y)\rangle \geqslant \mu\|x-y\|^{2}, \quad \forall x, y \in R^{n} .
$$

Definition 2.3 ([34]). Let $\Omega=[0, T] \times R^{n}$. A mapping $G: \Omega \longrightarrow R^{n}$ (resp., $B: \Omega \rightarrow R^{m \times n}$ ) is said to be Lipschitz continuous if there exists a constant $L_{G}>0$ (resp., $L_{B}>0$ ) such that, for any $\left(t_{1}, x\right),\left(t_{2}, y\right) \in \Omega$,

$$
\left\|G\left(t_{1}, x\right)-G\left(t_{2}, y\right)\right\| \leqslant L_{G}\left(\left|t_{1}-t_{2}\right|+\|x-y\|\right), \quad\left(\text { resp., }\left\|B\left(t_{1}, x\right)-B\left(t_{2}, y\right)\right\| \leqslant L_{B}\left(\left|t_{1}-t_{2}\right|+\|x-y\|\right)\right) .
$$

Definition 2.4 ([17]). Let $\mathrm{H}$ be a real Hilbert space, $\mathrm{K}: \mathrm{H} \rightarrow 2^{\mathrm{H}}$ be a set-valued mapping such that $\mathrm{K}(\mathrm{u})$ is a closed and convex subset of $\mathrm{H}$. We say that $\mathrm{P}_{\mathrm{K}}: \mathrm{H} \rightarrow \mathrm{H}$ is a generalized f-projection operator if

$$
\mathrm{P}_{\mathrm{K}(\mathrm{x})} z=\arg \inf _{\xi \in \mathrm{K}(\mathrm{x})}\left\{\|z\|^{2}-2\langle z, \xi\rangle+\|\xi\|^{2}\right\}, \quad \forall z \in \mathrm{H} .
$$

Definition 2.5 ([1]). Let $X, Y$ be two metric spaces. Consider a set-valued mapping $F: X \rightarrow Y$ with nonempty images. A single-valued mapping $f: X \rightarrow Y$ is called a selection of $F$ if, for all $x \in X, f(x) \in F(x)$.

As the definition of the Carathéodory weak solution for the differential variational inequality in [20], we can define similarly the Carathéodory weak solution for the DIQVI (1.2) as follows.

Definition 2.6. Let $x(t), u(t):[0, T] \rightarrow R^{n}$ be two functions. $(x(t), u(t))$ is called a Carathéodory weak solution of the DIQVI (1.2) if $x(t)$ is absolutely continuous on $[0, T]$ and satisfies the differential equation for almost all $t \in[0, T], u(t)$ is an integrable function on $[0, T]$ and satisfies the inverse quasi-variational inequality for almost all $t \in[0, T]$.

Lemma 2.7 ([3]). Let $\mathrm{N}$ be a nonempty compact convex subset of $\mathrm{R}^{\mathrm{n}}$ and $\phi: \mathrm{N} \rightarrow \mathrm{N}$ be a continuous mapping. Then $\phi$ has a fixed point in $\mathrm{N}$.

Lemma 2.8 ([1]). Let $\mathrm{X}$ be a metric space, $\mathrm{Y}$ be a Banach space, and $\mathrm{P}: \mathrm{X} \rightrightarrows \mathrm{Y}$ be a lower semi-continuous set-valued mapping with closed convex values. Then there exists a continuous selection $\mathrm{g}: \mathrm{X} \rightarrow \mathrm{Y}$ of $\mathrm{P}$.

The following two lemmas from the reference [20] are crucial to prove the main results in Sections 3 and 4 of this paper. 
Lemma 2.9. Let $\mathbb{F}: \Omega \rightrightarrows \mathrm{R}^{\mathrm{m}}$ be an upper semi-continuous set-valued mapping with nonempty closed convex values. Suppose that there exists a scalar $\rho_{\mathbb{F}}>0$ satisfying

$$
\sup \{\|y\|: y \in \mathbb{F}(t, x)\} \leqslant \rho_{\mathbb{F}}(1+\|x\|), \quad \forall(t, x) \in \Omega .
$$

Then, for every $x^{0} \in R^{n}$, the differential inclusion (DI) $\dot{x} \in \mathbb{F}(t, x), x(0)=x^{0}$ has a weak solution in the sense of Carathéodory.

Lemma 2.10. Let $\mathrm{h}: \Omega \times \mathrm{R}^{\mathrm{m}} \rightarrow \mathrm{R}^{\mathrm{m}}$ be a continuous function and $\mathrm{U}: \Omega \rightrightarrows \mathrm{R}^{\mathrm{n}}$ be a closed set-valued mapping such that for some constant $\eta_{\mathrm{u}}>0$,

$$
\sup _{\mathfrak{u} \in \mathrm{u}(\mathrm{t}, x)}\|\mathrm{u}\| \leqslant \eta_{u}(1+\|x\|), \quad \forall(t, x) \in \Omega .
$$

Let $v:[0, T] \rightarrow R^{m}$ be a measurable function and $x:[0, T] \rightarrow R^{m}$ be a continuous function satisfying $v(t) \in$ $\mathrm{h}(\mathrm{t}, \mathrm{x}(\mathrm{t}), \mathrm{U}(\mathrm{t}, \mathrm{x}(\mathrm{t})))$ for almost all $\mathrm{t} \in[0, \mathrm{~T}]$. Then, there exists a measurable function $\mathrm{u}:[0, \mathrm{~T}] \rightarrow \mathrm{R}^{\mathrm{n}}$ such that $\mathrm{u}(\mathrm{t}) \in \mathrm{U}(\mathrm{t}, \mathrm{x}(\mathrm{t}))$ and $v(\mathrm{t})=\mathrm{h}(\mathrm{t}, \mathrm{x}(\mathrm{t}), \mathrm{u}(\mathrm{t}))$ for almost all $\mathrm{t} \in[0, \mathrm{~T}]$.

\section{Existence of Carathéodory weak solutions to the DIQVI}

In this section, based on Lemmas 2.9 and 2.10, we give several theorems on the existence of Carathéodory weak solutions to the DIQVI (1.2) under different conditions.

We firstly define a set-valued mapping $\mathbb{F}(t, x): \Omega \rightarrow 2^{R^{m}}$ as follows:

$$
\mathbb{F}(t, x)=\{f(t, x)+B(t, x) u: u \in \operatorname{SIQVI}(h(\cdot), G(t, x)+F(\cdot), K(\cdot))\}
$$

Then we make the following hypotheses for the DIQVI (1.2) considered:

(A) $f, B$, and $G$ are Lipschitz continuous functions on $\Omega$ with Lipschitz constants $\rho_{f}>0, \rho_{B}>0$, and $\rho_{\mathrm{G}}>0$, respectively.

(B) B and $f$ are bounded on $\Omega$ with

$$
\sigma_{B}=\sup _{(t, x) \in \Omega}\|B(t, x)\|<\infty, \quad \sigma_{f}=\sup _{(t, x) \in \Omega}\|f(t, x)\|<\infty,
$$

respectively.

Lemma 3.1. Let ( $f, G, B)$ satisfy the conditions (A) and (B), $h, F: R^{n} \rightarrow R^{n}$ be two continuous functions, and the set-valued mapping $\mathrm{K}: \mathrm{R}^{\mathrm{n}} \rightarrow 2^{\mathrm{R}^{\mathrm{n}}}$ be continuous. Suppose that, for all $\mathrm{q} \in \mathrm{G}(\Omega)$ with $\mathrm{G}(\Omega)$ being the range of the function $\mathrm{G}$ on $\Omega$, the solution set of the inverse quasi-variational in the equality $\operatorname{SIQVI}(\mathrm{h}(\cdot), \mathrm{q}+\mathrm{F}(\cdot), \mathrm{K}(\cdot))$ is nonempty and there exists a constant $\rho>0$ such that

$$
\sup \{\|u\|: u \in \operatorname{SIQVI}(h(\cdot), q+F(\cdot), K(\cdot))\} \leqslant \rho(1+\|q\|) .
$$

Then there exists a constant $\rho^{\mathbb{F}}>0$ such that (2.1) holds for the set-valued mapping $\mathbb{F}$ defined by (3.1) and $\mathbb{F}$ is upper semi-continuous and thus with closed value on $\Omega$.

Proof. Since $f$ and $G$ are Lipschitz continuous on $\Omega$ with Lipschitz constants $\rho_{f}>0$ and $\rho_{G}>0$, we deduce that, for all $(t, x) \in \Omega$,

$$
\|f(t, x)\| \leqslant \rho_{f}(1+\|x\|)
$$

and

$$
\|\mathrm{G}(\mathrm{t}, x)\| \leqslant \rho_{\mathrm{G}}(1+\|x\|) .
$$

In a similar way of Lemma 6.2 in [20], it follows from (3.2), (3.3), and (3.4) that there exists $\rho^{\mathbb{F}}>0$ such that (2.1) holds. 
Now, we prove the upper semi-continuity of $\mathbb{F}$ on $\Omega$. It suffices to show that the set-valued mapping $\mathbb{F}$ is closed on $\Omega$ with the condition (2.1) (see [20]). To this end, let the sequence $\left\{\left(t_{n}, x_{n}\right)\right\} \subset \Omega$ be a sequence converging to some vector $\left(t_{0}, x_{0}\right) \in \Omega$ and $\left\{f\left(t_{n}, x_{n}\right)+B\left(t_{n}, x_{n}\right) u_{n}\right\}$ converge to some vector $z_{0} \in R^{m}$ as $n \rightarrow \infty$, where $u_{n} \in \operatorname{SIQVI}\left(h(\cdot), G\left(t_{n}, x_{n}\right)+F(\cdot), K(\cdot)\right)$ for every $n \geqslant 1$. Thus

$$
G\left(t_{n}, x_{n}\right)+F\left(u_{n}\right) \in K\left(u_{n}\right)
$$

and

$$
\left\langle h\left(u_{n}\right), y-G\left(t_{n}, x_{n}\right)-F\left(u_{n}\right)\right\rangle \geqslant 0, \quad \forall y \in K\left(u_{n}\right) .
$$

By the condition (3.2), the sequence $\left\{u_{n}\right\}$ is bounded and thus there exists a convergent subsequence denoted by $\left\{u_{n_{i}}\right\}$ with a limit $u_{0} \in R^{n}$. Since $G$ is Lipschitz continuous on $\Omega, F$ is continuous on $R^{n}$ and $\mathrm{K}$ is upper semi-continuous on $\mathrm{R}^{\mathrm{n}}$, it follows from (3.5) that

$$
\mathrm{G}\left(\mathrm{t}_{0}, \mathrm{x}_{0}\right)+\mathrm{F}\left(\mathrm{u}_{0}\right) \in \mathrm{K}\left(\mathrm{u}_{0}\right) .
$$

The lower semi-continuity of $K$ implies that for any $\hat{y} \in K\left(u_{0}\right)$, there exists $\hat{y}_{n} \in K\left(u_{n}\right)$ such that $\hat{y}_{n} \rightarrow \hat{y}$. This together with (3.6) implies that

$$
\left\langle h\left(u_{0}\right), \hat{y}-G\left(t_{0}, x_{0}\right)-F\left(u_{0}\right)\right\rangle \geqslant 0, \quad \forall \hat{y} \in K\left(u_{0}\right) .
$$

Thus,

$$
f\left(t_{n}, x_{n}\right)+B\left(t_{n}, x_{n}\right) u_{n} \longrightarrow z_{0}=f\left(t_{0}, x_{0}\right)+B\left(t_{0}, x_{0}\right) u_{0} \in \mathbb{F}\left(t_{0}, x_{0}\right),
$$

which implies that $\mathbb{F}$ is closed on $\Omega$. This completes the proof.

Now, we are in position to prove a theorem on the existence of the Carathéodory weak solutions for the DIQVI (1.2) based on Lemma 3.1.

Theorem 3.2. Let ( $\mathrm{f}, \mathrm{G}, \mathrm{B})$ satisfy the conditions $(\mathrm{A})$ and $(\mathrm{B}), \mathrm{h}, \mathrm{F}: \mathrm{R}^{\mathrm{n}} \rightarrow \mathrm{R}^{\mathrm{n}}$ be Lipschitz continuous functions with Lipschitz constants $\alpha$ and $\beta$, respectively, $h$ be strongly monotone with constant $\lambda$ on $R^{n}$, and $(F, h)$ be a $\mu$-strongly monotone couple on $\mathrm{R}^{\mathrm{n}}$. Assume that $\mathrm{K}: \mathrm{R}^{\mathrm{n}} \rightarrow 2^{\mathrm{R}^{\mathrm{n}}}$ is a continuous set-valued mapping such that $\mathrm{K}(\mathrm{u}) \subset \mathrm{R}^{\mathfrak{n}}$ is a closed convex set for each $\mathrm{u} \in \mathrm{R}^{\mathrm{n}}$. Then, under the following three conditions:

(i) there exists $\mathrm{k}>0$ such that $\left\|\mathrm{P}_{\mathrm{K}(\mathrm{u})} z-\mathrm{P}_{\mathrm{K}(\mathrm{y})} z\right\| \leqslant \mathrm{k}\|\mathrm{u}-\mathrm{y}\|$ for all $\mathrm{u}, \mathrm{y} \in \mathrm{R}^{\mathrm{n}}$ and $z \in\{e: e=\mathrm{q}+\mathrm{F}(\mathrm{u})-$ $\left.h(u), u \in R^{n}, q \in G(\Omega)\right\} ;$

(ii) there exists $\rho>0$ such that (3.2) holds for all $\mathrm{q} \in \mathrm{G}(\Omega)$;

(iii) $\left(\beta^{2}-2 \mu+\alpha^{2}\right)^{1 / 2}+\left(1-2 \lambda+\alpha^{2}\right)^{1 / 2}<1-k$;

the DIQVI (1.2) admits at least a Carathéodory weak solution.

Proof. By the assumptions (i) and (iii), it follows from Theorem 4.1 in [17] that the solution set $\operatorname{SIQVI}(h(\cdot), q+F(\cdot), K(\cdot))$ is a nonempty singleton for every $q \in G(\Omega)$. Then, by Lemmas 2.9 and 3.1, the differential inclusion DI $: \dot{x} \in \mathbb{F}(t, x), x(0)=\chi^{0}$ has a Carathéodory weak solution. Similar to the proof of Proposition 6.1 of [20], it is easy to prove that the DIQVI (1.2) admits a Carathéodory weak solution by using Lemma 2.10. This completes the proof.

Remark 3.3. If the mapping $\mathrm{h}=\mathrm{I}$ (the identity mapping) and the set $\mathrm{K}(\mathrm{x})$ is a constant set $\mathrm{K}$ on $\mathrm{R}^{\mathrm{n}}$, then the DIQVI (1.2) reduces to the differential inverse variational inequality in [16]. Therefore, Theorem 3.2 generalizes some results of Theorem 3.2 in [16].

Remark 3.4. As we see in the proof of Theorem 3.2, the conditions (i) and (iii) in Theorem 3.2 guarantee the uniqueness of solution to the IQVI $(h(\cdot), q+F(\cdot), K(\cdot))$. 
Next, we prove the existence of the Carathéodory weak solution for the DIQVI (1.2) without the uniqueness of solution to the IQVI $(h(\cdot), q+F(\cdot), K(\cdot))$.

Lemma 3.5. Let $h, F: R^{n} \rightarrow R^{n}$ and $P_{K(\cdot)}(q+F(\cdot)-h(\cdot))$ be continuous functions on $R^{n}$ for all $\mathrm{q} \in \mathrm{G}(\Omega)$, and $\mathrm{K}: \mathrm{R}^{\mathrm{n}} \rightarrow 2^{\mathrm{R}^{\mathrm{n}}}$ be a set-valued mapping such that, for each $\mathrm{u} \in \mathrm{R}^{\mathrm{n}}, \mathrm{K}(\mathrm{u}) \subset \mathrm{R}^{\mathrm{n}}$ is a closed convex set. Assume that $\mathrm{N}$ is a nonempty compact convex subset of $\mathrm{R}^{\mathrm{n}}$ such that $\phi(\cdot)=\mathrm{I}(\cdot)-(\mathrm{q}+\mathrm{F}(\cdot))+\mathrm{P}_{\mathrm{K}(\cdot)}(\mathrm{q}+\mathrm{F}(\cdot)-\mathrm{h}(\cdot))$ is a mapping from $\mathrm{N}$ to $\mathrm{N}$. Then the solution set $\operatorname{SIQVI}(\mathrm{h}(\cdot), \mathrm{q}+\mathrm{F}(\cdot), \mathrm{K}(\cdot))$ of the $\operatorname{IQVI}(\mathrm{h}(\cdot), \mathrm{q}+\mathrm{F}(\cdot), \mathrm{K}(\cdot))$ is nonempty.

Proof. Since $h, F: R^{n} \rightarrow R^{n}$ and $P_{K(\cdot)}(q+F(\cdot)-h(\cdot))$ are continuous on $R^{n}, \phi$ is also continuous on $N$. By Lemma 2.7, the mapping $\phi$ has a fixed point in $N$. This implies that there exists $u \in N$ such that

$$
u=\phi(u)=u-(q+F(u))+P_{k(u)}(q+F(u)-h(u))
$$

and thus

$$
q+F(u)=P_{K(u)}(q+F(u)-h(u)),
$$

which implies that $q+F(u) \in K(u)$. Define a function $j:[0,1] \rightarrow R$ as follows:

$$
\mathfrak{j}(\lambda)=\langle\lambda(q+F(u))+(1-\lambda) \hat{y}-(q+F(u))+h(u), \lambda(q+F(u))+(1-\lambda) \hat{y}-(q+F(u))+h(u)\rangle
$$

for all $\lambda \in[0,1]$, where $\hat{y}$ is a given point on $K(u)$. By $(3.7), j$ gets its minimum at $\lambda=1$. It follows from $j^{\prime}(1)=0$ that

$$
\langle h(u), \hat{y}-q-F(u)\rangle \geqslant 0, \quad \forall \hat{y} \in K(u) .
$$

This together with (3.7) shows that $u \in \operatorname{SIQVI}(h(\cdot), q+F(\cdot), K(\cdot))$ and the solution set SIQVI(h( $)$, $q+$ $\mathrm{F}(\cdot), \mathrm{K}(\cdot))$ of the IQVI $(\mathrm{h}(\cdot), \mathrm{q}+\mathrm{F}(\cdot), \mathrm{K}(\cdot))$ is nonempty. This completes the proof.

Theorem 3.6. Let ( $\mathrm{f}, \mathrm{G}, \mathrm{B})$ satisfy the conditions (A) and (B) and $\mathrm{K}: \mathrm{R}^{\mathrm{n}} \rightarrow 2^{\mathrm{R}^{\mathrm{n}}}$ be a continuous set-valued mapping. Assume that the conditions in Lemma 3.5 hold. Then, under the following conditions:

(i) $\operatorname{SIQVI}(\mathrm{h}(\cdot), \mathrm{q}+\mathrm{F}(\cdot), \mathrm{K}(\cdot))$ is a convex set for all $\mathrm{q} \in \mathrm{G}(\Omega)$;

(ii) there exists $\rho>0$ such that (3.2) holds for all $\mathrm{q} \in \mathrm{G}(\Omega)$;

the DIQVI (1.2) admits a Carathéodory weak solution.

Proof. By Lemma 3.5, the SIQVI $(\mathrm{h}(\cdot), \mathrm{q}+\mathrm{F}(\cdot), \mathrm{K}(\cdot))$ is a nonempty set.

Next, we show that $\operatorname{SIQVI}(h(\cdot), q+F(\cdot), K(\cdot))$ is closed. In fact, let $\left\{u_{n}\right\} \subset \operatorname{SIQVI}(h(\cdot), q+F(\cdot), K(\cdot))$ with $u_{n} \rightarrow u_{0}$, which means that

$$
q+F\left(u_{n}\right) \in K\left(u_{n}\right)
$$

and

$$
\left\langle h\left(u_{n}\right), y-q-F\left(u_{n}\right)\right\rangle \geqslant 0, \quad \forall y \in K\left(u_{n}\right) .
$$

Since $\mathrm{F}$ and $\mathrm{K}$ are continuous, it follows that

$$
q+F\left(u_{0}\right) \in K\left(u_{0}\right) .
$$

Similar to the proof in Lemma 3.1, we can obtain that

$$
\left\langle\mathrm{h}\left(\mathrm{u}_{0}\right), \mathrm{y}-\mathrm{q}-\mathrm{F}\left(\mathrm{u}_{0}\right)\right\rangle \geqslant 0, \quad \forall \mathrm{y} \in \mathrm{K}\left(\mathrm{u}_{0}\right),
$$

which implies that $u_{0} \in \operatorname{SIQVI}(h(\cdot), q+F(\cdot), K(\cdot))$ and thus the $\operatorname{SIQVI}(h(\cdot), q+F(\cdot), K(\cdot))$ is nonempty closed and convex. Moreover, from Lemma 3.1, there exists a constant $\rho^{\mathbb{F}}>0$ such that (2.1) holds and $\mathbb{F}$ is upper semicontinuous and closed-valued on $\Omega$. Therefore, by applying Lemmas 2.9 and 2.10, the DIQVI (1.2) admits a Carathéodory weak solution. This completes the proof. 
Remark 3.7. As suggested by the referee, it is important to consider that under what conditions the conditions (i) and (ii) in Theorem 3.2 hold.

The following lemma gives some conditions under which the condition (ii) in Theorem 3.2 holds.

Lemma 3.8. Let $\mathrm{F}: \mathrm{R}^{\mathrm{n}} \rightarrow \mathrm{R}^{\mathrm{n}}$ be a bounded, continuous, and inverse linear mapping with $\|\mathrm{F}\|>\mathrm{c}$ and $\mathrm{K}: \mathrm{R}^{\mathrm{n}} \rightarrow$ $2^{R^{n}}$ be a set-valued mapping which satisfies linear growth, i.e., there exists a constant $c>0$ such that

$$
\sup _{v \in K(w)}\|v\| \leqslant c(1+\|w\|), \quad \forall w \in \mathrm{R}^{\mathrm{n}} .
$$

Suppose that the set $\operatorname{SIQVI}(\mathrm{h}(\cdot), \mathrm{q}+\mathrm{F}(\cdot), \mathrm{K}(\cdot))$ is nonempty for all $\mathrm{q} \in \mathrm{G}(\Omega)$. Then there exists $\rho>0$ such that (3.2) holds for all $\mathrm{q} \in \mathrm{G}(\Omega)$.

Proof. Since the $\operatorname{SIQVI}(\mathrm{h}(\cdot), \mathrm{q}+\mathrm{F}(\cdot), \mathrm{K}(\cdot))$ is nonempty for all $\mathrm{q} \in \mathrm{G}(\Omega)$, we deduce that

$$
q+F(u) \in K(u)
$$

for any $q \in G(\Omega)$ and $u \in \operatorname{SIQVI}(h(\cdot), q+F(\cdot), K(\cdot))$. By the linear growth of the mapping $K$, it follows that

$$
\|\mathrm{q}+\mathrm{F}(\mathrm{u})\| \leqslant \mathrm{c}(1+\|\mathrm{u}\|), \quad \forall \mathrm{q} \in \mathrm{G}(\Omega)
$$

which implies that

$$
\|\mathrm{u}\|=\left\|\mathrm{F}^{-1} \mathrm{~F}(\mathrm{u})\right\| \leqslant\left\|\mathrm{F}^{-1}\right\|[\mathrm{c}(1+\|\mathrm{u}\|)+\|\mathrm{q}\|] .
$$

Since $F$ is a bounded continuous linear mapping, $F^{-1}$ is bounded. Thus, by the condition $\|F\|>c$, we obtain that

$$
\left(1-\left\|\mathrm{F}^{-1} \mathrm{c}\right\|\right)\|\mathrm{u}\| \leqslant \mathrm{c}\left\|\mathrm{F}^{-1}\right\|+\left\|\mathrm{F}^{-1}\right\|\|\mathrm{q}\|, \quad \forall \mathrm{q} \in \mathrm{G}(\Omega),
$$

which implies that

$$
\|\mathrm{u}\| \leqslant \frac{\max \left\{\mathrm{c}\left\|\mathrm{F}^{-1}\right\|,\left\|\mathrm{F}^{-1}\right\|\right\}}{1-\left\|\mathrm{F}^{-1} \mathrm{c}\right\|}(1+\|\mathrm{q}\|), \quad \forall \mathrm{q} \in \mathrm{G}(\Omega)
$$

This means that, for all $\mathrm{q} \in \mathrm{G}(\Omega)$, (3.2) holds with $\rho=\frac{\max \left\{\mathrm{c}\left\|\mathrm{F}^{-1}\right\|,\left\|\mathrm{F}^{-1}\right\|\right\}}{1-\left\|\mathrm{F}^{-1} \mathrm{c}\right\|}(1+\|\mathrm{q}\|)$.

\section{Euler computation method to the DIQVI}

In this section, by the Euler computation method used in the [20], we construct an Euler timedependent scheme for solving the DIQVI (1.2). By using Lemmas 2.9 and 2.10, we prove a convergence result on the Euler time-dependent scheme. In what follows we denote by $\mathrm{L}^{2}[0, T]$ the set of all measurable functions $u:[0, T] \rightarrow R^{n}$ satisfying $\int_{0}^{T}\|u(t)\|^{2} d t<\infty$, in which the inner product is defined as

$$
\langle u, v\rangle=\int_{0}^{T}\langle u(t), v(t)\rangle d t, \quad \forall u, v \in \mathrm{L}^{2}[0, \mathrm{~T}] .
$$

Similar to the Euler time-dependent scheme for the differential variational inequality considered in [20] by Pang and Stewart, we can construct the Euler time-dependent scheme for the DIQVI (1.2) as follows.

Choose an equidistant grid $0=t_{0}<t_{1}<\cdots<t_{N}=T$ with step-size $l=\frac{T}{N}$. Setting $x^{l, 0}=x_{0}$, we compute iterates $\left\{x^{l, 1}, x^{l, 2}, \ldots, x^{l, i}, \ldots, x^{l, N_{l}}\right\} \subset R^{m}$ and $\left\{u^{l, 1}, u^{l, 2}, \ldots, u^{l, i}, \ldots, u^{l, N_{l}}\right\} \subset R^{n}$ as follows: for $i=0,1, \cdots, N_{l}$,

$$
\left\{\begin{array}{l}
x^{l, i+1}=x^{l, i}+l\left[f\left(t_{l, i+1}, \theta x^{l, i}+(1-\theta) x^{l, i+1}\right)+B\left(t_{l, i}, x^{l, i}\right) u^{l, i+1}\right] \\
u^{l, i+1} \in \operatorname{SIQVI}\left(h(\cdot), G\left(t_{l, i+1}, x^{l, i+1}\right)+F(\cdot), K(\cdot)\right)
\end{array}\right.
$$


where $N_{l}=\frac{T}{l}-1, \theta \in[0,1]$. The existence of $\left(\chi^{l, i+1}, u^{l, i+1}\right)$ satisfying (4.1) can be obtained in a similar way as Proposition 7.1 in [20]. With the consequences $\left\{x^{l, i+1}\right\}$ and $\left\{u^{l, i+1}\right\}$, we denote by $\hat{x}^{l}(\cdot)$ and $\hat{u}^{l}(\cdot)$ the continuous piecewise linear interpolant of the consequence $\left\{x^{l, i+1}\right\}$ and the constant piecewise interpolant of the consequence $\left\{u^{l, i+1}\right\}$, respectively. This implies that

$$
\begin{cases}\hat{x}^{l}(t)=x^{l, i}+\frac{t-t_{l, i}}{l}\left(x^{l, i+1}-x^{l, i}\right), & \forall t \in\left[t_{l, i}, t_{l, i+1}\right], \\ \hat{u}^{l}(t)=u^{l, i+1}, & \forall t \in\left(t_{l, i}, t_{l, i+1}\right]\end{cases}
$$

for each $i=0,1, \cdots, N_{l}$.

Now, we are in position to prove the convergence result on the Euler time-dependent scheme for solving the DIQVI (1.2), which is based on the following important lemma.

Lemma 4.1. Let ( $\mathrm{f}, \mathrm{B}, \mathrm{G})$ satisfy the conditions $(\mathrm{A})$ and $(\mathrm{B}), \mathrm{K}: \mathrm{R}^{\mathrm{n}} \rightarrow 2^{\mathrm{R}^{\mathrm{n}}}$ be a lower semi-continuous set-valued mapping on $\mathrm{R}^{\mathrm{n}}$ with nonempty closed and convex values, and $\mathrm{F}: \mathrm{L}^{2}[0, \mathrm{~T}] \rightarrow \mathrm{L}^{2}[0, \mathrm{~T}]$ be a continuous mapping. Assume that $\mathrm{x} \in \mathrm{C}\left([0, \mathrm{~T}] ; \mathrm{R}^{\mathrm{m}}\right)$ be a continuous vector function. If $\mathrm{u} \in \mathrm{L}^{2}[0, \mathrm{~T}]$ satisfies $\mathrm{G}(\mathrm{t}, \mathrm{x}(\mathrm{t}))+\mathrm{F}(\mathrm{u}(\mathrm{t})) \in$ $\mathrm{K}(\mathrm{u}(\mathrm{t}))$ for all $\mathrm{t} \in[0, \mathrm{~T}]$ and

$$
\int_{0}^{T}\langle h(u(t)), \tilde{u}(t)-G(t, x(t))-F(u(t))\rangle d t \geqslant 0, \quad \forall \tilde{u} \in L^{2}([0, T] ; K(u(t))
$$

then $\mathrm{u}(\mathrm{t}) \in \operatorname{SIQVI}(\mathrm{h}(\cdot), \mathrm{G}(\mathrm{t}, \mathrm{x}(\mathrm{t}))+\mathrm{F}(\cdot), \mathrm{K}(\cdot))$ for almost all $\mathrm{t} \in[0, \mathrm{~T}]$.

Proof. To prove this lemma, we assume that the contrary holds, which means there exists a subset $E$ of $[0, T]$ with $m(E)>0$ (where $m(E)$ denotes the Lebesgue measure of $E$ ) such that, for any $t \in E$,

$$
\mathrm{u}(\mathrm{t}) \notin \operatorname{SIQVI}(h(\cdot), \mathrm{G}(\mathrm{t}, x(\mathrm{t}))+\mathrm{F}(\cdot), \mathrm{K}(\cdot)) .
$$

By Lusin's theorem, for any $\xi>0$, there exists a closed subset $E_{1}$ of $E$ with $m\left(E \backslash E_{1}\right)<\xi$ such that $u(t)$ is continuous on $E_{1}$. Therefore, for any $\varepsilon>0$, there exists $t_{0} \in E_{1}$ and a neighborhood of $t_{0}$, denoted by $\mathrm{u}\left(\mathrm{t}_{0}\right)$, such that $\left\|\mathrm{u}(\mathrm{t})-\mathrm{u}\left(\mathrm{t}_{0}\right)\right\|<\varepsilon$ for all $\mathrm{t} \in \mathrm{U}\left(\mathrm{t}_{0}\right) \bigcap \mathrm{E}_{1}$ with $\mathrm{m}\left(\mathrm{U}\left(\mathrm{t}_{0}\right) \bigcap \mathrm{E}_{1}\right)>0$. Since $t_{0} \in \mathrm{E}_{1}$, there exists $\tilde{u}_{0} \in \mathrm{K}\left(\mathrm{u}\left(\mathrm{t}_{0}\right)\right)$ such that

$$
\left\langle h\left(u\left(t_{0}\right)\right), \tilde{u}_{0}-G\left(t_{0}, x\left(t_{0}\right)\right)-F\left(u\left(t_{0}\right)\right)\right\rangle=-C<0 .
$$

Let

$$
\tilde{S}(u)= \begin{cases}\tilde{u}_{0}, & u=u\left(t_{0}\right), \\ K(u), & u \neq u\left(t_{0}\right) .\end{cases}
$$

Since $\mathrm{K}$ is a lower semi-continuous set-valued mapping on $\mathrm{R}^{\mathrm{n}}$ with nonempty closed and convex values and $\tilde{u}_{0} \in \mathrm{K}\left(\mathfrak{u}\left(\mathrm{t}_{0}\right)\right)$, it follows that $\tilde{S}$ is lower semi-continuous on $\mathrm{R}^{\mathrm{n}}$ with nonempty closed and convex values. By Lemma 2.8, there exists a continuous selection of $\tilde{S}$, which is denoted by $H$. Therefore, $\mathrm{H}\left(\mathrm{u}\left(\mathrm{t}_{0}\right)\right)=\tilde{\mathrm{u}}_{0}$ and $\mathrm{H}(\mathrm{u}(\mathrm{t}))$ is continuous on $\mathrm{U}\left(\mathrm{t}_{0}\right) \cap \mathrm{E}_{1}$. Let

$$
g(t)=\langle h(u(t)), H(u(t))-G(t, x(t))+F(u(t))\rangle,
$$

which is continuous on $\mathrm{U}\left(\mathrm{t}_{0}\right) \bigcap \mathrm{E}_{1}$. From (4.4), $\mathrm{g}\left(\mathrm{t}_{0}\right)=-\mathrm{C}<0$. The continuity of $\mathrm{g}$ implies that there exists a closed neighborhood $U\left(t_{0}, \delta\right)$ of $t_{0}$ such that, for all $t \in E_{2}=U\left(t_{0}, \delta\right) \cap E_{1}$ with $m\left(E_{2}\right)>0$,

$$
\left\|g(t)-g\left(t_{0}\right)\right\| \leqslant \frac{C}{2} \text {. }
$$

Thus it follows that

$$
g(t) \leqslant g\left(t_{0}\right)+\frac{C}{2}=-\frac{C}{2}, \quad \forall t \in E_{2}
$$

and

$$
\int_{E_{2}}\langle h(u(t)), H(u(t))-G(t, x(t))-F(u(t))\rangle d t=\int_{E_{2}} g(t) d t<0 .
$$


Let

$$
\tilde{u}(t)= \begin{cases}H(u(t)), & t \in E_{2}, \\ G(t, x(t))+F(u(t)), & t \in[0, T] \backslash E_{2} .\end{cases}
$$

It is clear that $\tilde{u} \in\left(\mathrm{L}^{2}([0, \mathrm{~T}], \mathrm{K}(\mathrm{u}(\mathrm{t})))\right.$. Thus we have

$$
\begin{aligned}
\int_{0}^{T}\langle h(u(t)), \tilde{u}(t)-G(t, x(t))-F(u(t))\rangle d t= & \int_{E_{2}}\langle h(u(t)), H(u(t))-G(t, x(t))-F(u(t))\rangle d t \\
& +\int_{[0, T] \backslash E_{2}}\langle h(u(t)), G(t, x(t))+F(u(t))-G(t, x(t))-F(u(t))\rangle d t \\
= & \int_{E_{2}}\langle h(u(t)), H(u(t))-G(t, x(t))-F(u(t))\rangle d t<0,
\end{aligned}
$$

which contradicts (4.3). This completes the proof.

Theorem 4.2. Let $(\mathrm{f}, \mathrm{B}, \mathrm{G})$ satisfy the conditions (A) and (B), $\mathrm{h}: \mathrm{R}^{\mathrm{n}} \rightarrow \mathrm{R}^{\mathrm{n}}$ be a continuous and bounded function on $\mathrm{R}^{\mathrm{n}}$, and $\mathrm{K}: \mathrm{R}^{\mathrm{n}} \rightarrow 2^{\mathrm{R}^{n}}$ be a continuous set-valued mapping on $\mathrm{R}^{\mathrm{n}}$ with nonempty closed and convex values and $\mathrm{K}$ has a linear growth. Suppose that, for any $\mathrm{q} \in \mathrm{G}(\Omega)$, $\operatorname{SIQVI}(\mathrm{h}(\cdot), \mathrm{q}+\mathrm{F}(\cdot), \mathrm{K}(\cdot)) \neq \emptyset$, there exists a constant $\rho>0$ such that (3.2) holds. Then every sequence pair $\left\{\left(\hat{x}^{l}, \hat{u}^{l}\right)\right\}$ defined by (4.2) has a subsequence pair $\left\{\left(\hat{x}^{l_{v}}, \hat{u}^{l_{v}}\right)\right\}$ such that $\hat{x}^{l_{v}} \rightarrow \tilde{x}$ uniformly in $[0, \mathrm{~T}]$ and $\hat{u}^{\mathrm{l}_{v}} \rightarrow \tilde{\mathrm{u}}$ weakly in $\mathrm{L}^{2}([0, \mathrm{~T}])$, as $v \rightarrow \infty$. Furthermore, suppose that $\mathrm{g}$ is a function such that $\mathrm{g}\left(\mathrm{u}_{\mathrm{n}}\right) \in \mathrm{K}\left(\mathrm{u}_{\mathrm{n}}\right), \mathrm{g}\left(\mathrm{u}_{\mathrm{n}}\right)$ uniformly converges to $\mathrm{g}(\mathrm{u})$, and $\mathrm{u}_{\mathrm{n}}$ weakly converges to $\mathrm{u}$, then $\mathrm{g}(\mathrm{u}) \in \mathrm{K}(\mathrm{u})$. Assume that $\mathrm{F}(\mathrm{u})=\psi(\mathrm{Eu})$, where $\psi: \mathrm{R}^{\mathrm{n}} \rightarrow \mathrm{R}^{\mathrm{n}}$ is Lipschitz continuous bounded and $\mathrm{E} \in \mathrm{R}^{\mathrm{n} \times \mathrm{n}}$ with the property that there exists a constant $\mathrm{C}>0$ such that, for all sufficiently small $\mathrm{l}>0$,

$$
\left\|E u^{l, i+1}-E u^{l, i}\right\| \leqslant l C .
$$

Then $(\tilde{x}, \tilde{u})$ is a Carathéodory weak solution of the DIQVI (1.2).

Proof. Since (f, B, G) satisfies the conditions (A) and (B) and there exists a constant $\rho>0$ such that (3.2) holds, we obtain, by Lemma 7.2 in [20], that there exists $0<h_{0}<\min \left\{\frac{1}{(1-\theta) L_{f}}, \frac{1}{(1-\theta) \rho_{f}}\right\}$ such that for all sufficiently small $l \in\left(0, h_{0}\right]$,

$$
\left\|x^{l, i+1}-x^{l, i}\right\| \leqslant \frac{l \rho_{f}\left(1+\left\|x^{l, i}\right\|+l \sigma_{B}\left\|u^{l, i+1}\right\|\right)}{1-l(1-\theta) \rho_{f}}
$$

where $L_{f}$ is Lipschitz constant of the function $f(t, \cdot)$ for all $t \in[0, T]$. Moreover, it follows from Lemma 7.3 in [20] that there exist constants $c_{0, x}, c_{1, x}, c_{0, u}, c_{1, u}$ and positive constant $l_{1}>0$ such that, for any $l \in\left(0, l_{1}\right]$ and $i=0,1, \cdots, N_{l}$,

$$
\left\{\begin{array}{l}
\left\|x^{l, i+1}\right\| \leqslant c_{0, x}+c_{1, x}\left\|x_{0}\right\| \\
\left\|u^{l, i+1}\right\| \leqslant c_{0, u}+c_{1, u}\left\|x_{0}\right\|
\end{array}\right.
$$

Combining (4.5) and (4.6) we can deduce that there exists $L_{x_{0}}>0$, which is independent of $l$ but depends on $x_{0}$, such that

$$
\left\|x^{l, i+1}-x^{l, i}\right\| \leqslant L_{x_{0}} l .
$$

By (4.2), the piecewise interpolant $\hat{x}^{l}$ is Lipschitz continuous on $[0, T]$ and the Lipschitz constant is independent of $l$. And thus, there exists an $h_{0}>0$ such that $\left\{\hat{x}^{l}\right\}$ is an equicontinuous family of functions for all $l \in\left(0, h_{0}\right]$. Let

$$
\left\|\hat{x}^{\mathrm{l}}\right\|_{\mathrm{L}_{\infty}}=\sup _{\mathrm{t} \in[0, \mathrm{~T}]}\left\|\hat{\mathrm{x}}^{\mathrm{l}}(\mathrm{t})\right\| .
$$

By the Arzelá-Ascoli theorem, we can obtain that there is a subsequence $\left\{\hat{x}^{l_{v}}\right\}$ of $\left\{\hat{x}^{l}\right\}$ such that $\left\{\hat{x}^{l_{v}}\right\}$ converges in the $L_{\infty}$ norm to a function $\tilde{x}$ on $[0, T]$. On the other hand, from (3.2) we know that the family of piecewise constant interpolants $\left\{\hat{u}^{l}\right\}$ is uniformly bounded in the $\mathrm{L}_{\infty}$ norm, which implies by 
the reflexivity of the Banach space $L^{2}([0, T])$ that there exists a subsequence $\left\{\hat{u}^{l_{v}}\right\}$ of $\left\{\hat{u}^{l}\right\}$ converging weakly to $\tilde{u}$.

Now, we show in the next that $(\tilde{x}, \tilde{u})$ is a Carathéodory weak solution of the DIQVI (1.2). It is sufficient to prove the following three assertions by the definition.

(I) First, we prove that, for almost all $t \in[0, T]$,

$$
\tilde{\mathfrak{u}}(\mathrm{t}) \in \operatorname{SIQVI}(\mathrm{h}(\cdot), \mathrm{G}(\mathrm{t}, x(\mathrm{t}))+\psi(\mathrm{E}(\cdot)), \mathrm{K}(\cdot)) .
$$

By the proof of Theorem 7.1 in [20], it is easy to get that the sequence $\left\{G\left(t, \hat{x}^{l_{v}}\right)+\psi\left(E \hat{u}^{l_{v}}\right)\right\} \subset K\left(\hat{u}^{l_{v}}\right)$ uniformly converges to $G(t, \tilde{x})+\psi(E \tilde{u})$ and thus, by the assumption, $G(t, \tilde{x})+\psi(E \tilde{u}) \in K(\tilde{u})$. Moreover, for any $y \in\left(L^{2}[0, t], K(\tilde{u}(t))\right.$, there exists $y^{l_{v}}(t) \in K\left(\hat{u}^{l_{v}}(t)\right)$ such that $y^{l_{v}}(t) \rightarrow y(t)$ on $t \in[0, T]$. Since $K$ has a linear growth, there exists a constant $c>0$ such that, for any $u \in R^{n}$,

$$
\|\mathrm{K}(\mathrm{u})\|=\sup _{v \in \mathrm{K}(\mathrm{u})}\|v\| \leqslant \mathrm{c}(1+\|\mathrm{u}\|) .
$$

Thus, for all $y^{l_{v}}(t) \in K\left(\hat{u}^{l_{v}}(t)\right)$,

$$
\left\|y^{l_{v}}(t)\right\| \leqslant c\left(1+\left\|u^{l_{v}}(t)\right\|\right),
$$

which indicates that $\left\{y^{l}\right\}$ is uniformly bounded in the $L_{\infty}$ norm by the uniform boundedness of $\left\{\hat{u}^{l}\right\}$ in the $L_{\infty}$ norm. Since $h, \psi$ are bounded, it follows from Lebesgue control convergence theorem that, for any $y(t) \in K(\tilde{u}(t))$,

$$
\begin{aligned}
& \int_{0}^{T}\langle h(\tilde{u}(t)), y(t)-G(t, \tilde{x}(t))-\psi(E \tilde{u}(t))\rangle d t \\
& \quad=\lim _{v \rightarrow \infty} \int_{0}^{T}\left\langle h\left(\hat{u}^{l_{v}}(t)\right), y^{l_{v}}(t)-G\left(t, \hat{x}^{l_{v}}(t)\right)-\psi\left(E \hat{u}^{l_{v}}(t)\right)\right\rangle d t
\end{aligned}
$$

where $y^{l_{v}}(t) \in K\left(u^{l_{v}}(t)\right)$.

On the other hand, for any $y^{l} \in \mathrm{L}^{2}\left([0, \mathrm{~T}], \mathrm{K}\left(\hat{\mathrm{u}}^{\mathrm{l}}(\mathrm{t})\right)\right)$, we have

$$
\begin{aligned}
& \int_{0}^{T}\left\langle h\left(\hat{u}^{l}(t)\right), y^{l}(t)-G\left(t, \hat{x}^{l}(t)\right)-\psi\left(E \hat{u}^{l}(t)\right)\right\rangle d t \\
& =\sum_{i=0}^{N_{l}} \int_{t_{l, i}}^{t_{l, i+1}}\left\langle h\left(u^{l, i+1}\right), y^{l}(t)-G\left(t, \hat{x}^{l}(t)\right)-\psi\left(E u^{l, i+1}\right)\right\rangle d t \\
& =\sum_{i=0}^{N_{l}} \int_{t_{l, i}}^{t_{l, i+1}}\left\langle h\left(u^{l, i+1}\right), y^{l}(t)-G\left(t_{l, i+1}, x^{l, i+1}\right)-\psi\left(E u^{l, i+1}\right)\right\rangle d t \\
& \quad+\sum_{i=0}^{N_{l}} \int_{t_{l, i}}^{t_{l, i+1}}\left\langle h\left(u^{l, i+1}\right), G\left(t_{l, i+1}, x^{l, i+1}\right)-G\left(t, \hat{x}^{h}(t)\right)\right\rangle d t \\
& \geqslant l \sum_{i=0}^{N_{l}} \frac{1}{l} \int_{t_{l, i}}^{t_{l, i+1}}\left\langle h\left(u^{l, i+1}\right), y^{l}(t)-G\left(t_{l, i+1}, x^{l, i+1}\right)-\psi\left(E u^{l, i+1}\right)\right\rangle-T l^{2} \rho_{G}\|h\|\left(1+L_{x_{0}}\right)\left\|\hat{u}^{l}\right\|_{L^{\infty}}
\end{aligned}
$$

Since $\mathrm{K}$ is a continuous set-valued mapping with nonempty closed convex value, it is easy to show that

$$
\frac{1}{l} \int_{t_{l, i}}^{t_{l, i+1}} y^{l}(t) d t \in K\left(u^{l, i+1}\right),
$$

which together with $u^{l, i+1} \in \operatorname{SIQVI}\left(h(\cdot), G\left(t_{l, i+1}, x^{l, i+1}\right)+\psi(E(\cdot)), K(\cdot)\right)$ and (4.8) implies that

$$
\lim _{\mathrm{l} \rightarrow 0} \int_{0}^{T}\left\langle\mathrm{~h}\left(\hat{\mathrm{u}}^{\mathrm{l}}(\mathrm{t})\right), \mathrm{y}^{\mathrm{l}}(\mathrm{t})-\mathrm{G}\left(\mathrm{t}, \hat{\mathrm{x}}^{\mathrm{l}}(\mathrm{t})\right)-\psi\left(E \hat{u}^{\mathrm{l}}(\mathrm{t})\right), \hat{\mathrm{u}}^{\mathrm{l}}(\mathrm{t})\right\rangle \mathrm{dt} \geqslant 0, \quad \forall \mathrm{y}^{\mathrm{l}} \in \mathrm{L}^{2}\left([0, \mathrm{~T}], \mathrm{K}\left(\hat{\mathrm{u}}^{\mathrm{l}}(\mathrm{t})\right)\right) .
$$


Thus it follows from (4.7) and (4.9) that, for any $y \in \mathrm{L}^{2}([0, \mathrm{~T}], \mathrm{K}(\tilde{\mathrm{u}}(\mathrm{t})))$,

$$
\int_{0}^{T}\langle h(\tilde{u}(t)), y(t)-G(t, \tilde{x}(t))-\psi(E \tilde{u}(t))\rangle d t \geqslant 0
$$

which indicates by Lemma 4.1 that, for almost all $t \in[0, T]$,

$$
\tilde{u}(t) \in \operatorname{SIQVI}(h(\cdot), G(t, x(t))+\psi(E(\cdot)), K(\cdot)) .
$$

(II) Next, we show that $\tilde{\chi}(0)=x_{0}$. In fact, since $\hat{x}^{l}(0)=x_{0}$ for all $l>0$ sufficiently small and $\hat{x}^{l_{v}} \rightarrow \tilde{\chi}$ uniformly as $v \rightarrow \infty$, it is easy to get that $\tilde{x}(0)=x_{0}$.

(III) Finally, we prove that, for any $0 \leqslant s \leqslant t \leqslant T$,

$$
\tilde{x}(t)-\tilde{x}(s)=\int_{s}^{t}[f(\tau, \tilde{x}(\tau))+B(\tau, \tilde{x}(\tau)) \tilde{u}(\tau)] d \tau,
$$

which can be carried out by similar arguments as in the proof of Theorem 7.1 in [20]. Therefore, from the assertions (I)-(III), it follows that $(\tilde{x}, \tilde{u})$ is a Carathéodory weak solution of the DIQVI (1.2). This completes the proof.

Remark 4.3. It is well-known that there are few papers concerning the computational methods for differential inverse variational inequality and differential inverse quasi-variational inequality. The Euler timedependent scheme for the DIQVI (1.2) proposed in this paper provides a powerful modeling paradigm for solving differential inverse variational inequality, differential inverse quasi-variational inequality, and differential quasi-variational inequality.

\section{Acknowledgment}

Wei Li was supported by Opening Fund of Geomathematics Key Laboratory of Sichuan Province of China (scsxdz201606), Yi-bin Xiao was supported by the National Natural Science Foundation of China (11771067), the China Postdoctoral Science Foundation (2015T80967), the Applied Basic Project of Sichuan Province (2016JY0170), the Open Foundation of State Key Laboratory of Electronic Thin Films and Integrated Devices (KFJJ201611), the Key Program of Education Department of Sichuan Province (16ZA0007), and the Fundamental Research Funds for the Central Universities (ZYGX2015J098), Nan-jing Huang was supported by the National Natural Science Foundation of China (11671282), and Yeol Je Cho was supported by Basic Science Research Program through the National Research Foundation of Korea (NRF) funded by the Ministry of Science, ICT and future Planning (2014R1A2A2A01002100).

\section{References}

[1] J. P. Aubin, H. Frankowska, Set-valued analysis, Systems \& Control: Foundations \& Applications, Birkhäuser Boston, Inc., Boston, MA, (1990). 2.5, 2.8

[2] D. Aussel, R. Gupta, A. Mehra, Gap functions and error bounds for inverse quasi-variational inequality problems, J. Math. Anal. Appl., 407 (2013), 270-280. 1, 2.2

[3] F. F. Bonsall, Lectures on some fixed point theorems of functional analysis, Notes by K. B. Vedak, Tata Institute of Fundamental Research, Bombay, (1962). 2.7

[4] M. K. Çamlıbel, W. P. M. H. Heemels, A. J. van der Schaft, J. M. Schumacher, Switched networks and complementarity, Special issue on switching and systems, IEEE Trans. Circuits Systems I Fund. Theory Appl., 50 (2003), 1036-1046. 1

[5] X.-J. Chen, S. Mahmoud, Implicit Runge-Kutta methods for Lipschitz continuous ordinary differential equations, SIAM J. Numer. Anal., 46 (2008), 1266-1280.

[6] X.-J. Chen, S.-H. Xiang, Newton iterations in implicit time-stepping scheme for differential linear complementarity systems, Math. Program., 138 (2013), 579-606. 1 
[7] I. Ekeland, R. Témam, Convex analysis and variational problems, Translated from the French, Corrected reprint of the 1976 English edition, Classics in Applied Mathematics, Society for Industrial and Applied Mathematics (SIAM), Philadelphia, PA, (1999). 1

[8] Y. Han, N.-J. Huang, J. Lu, Y.-B. Xiao, Existence and stability of solutions to inverse variational inequality problems, Appl. Math. Mech., 38 (2017), 749-764. 1, 1

[9] B.-S. He, X.-Z. He, H. X. Liu, Solving a class of constrained 'black-box' inverse variational inequalities, European J. Oper. Res., 204 (2010), 391-401. 1

[10] B. S. He, H. X. Liu, Inverse variational inequalities in economics-applications and algorithms, Sciencepaper Online, (2006). 1

[11] X.-Z. He, H. X. Liu, Inverse variational inequalities with projection-based solution methods, European J. Oper. Res., 208 (2011), 12-18. 1

[12] B. S. He, H. X. Liu, M. Li, X. Z. He, PPA-based methods for monotone inverse variational inequalities, Sciencepaper Online, (2006).

[13] R. Hu, Y.-P. Fang, Well-posedness of inverse variational inequalities, J. Convex Anal., 15 (2008), 427-437. 1

[14] X.-S. Li, N.-J. Huang, D. O'Regan, Differential mixed variational inequalities in finite dimensional spaces, Nonlinear Anal., 72 (2010), 3875-3886. 1

[15] X. Li, X.-S. Li, N.-J. Huang, A generalized f-projection algorithm for inverse mixed variational inequalities, Optim. Lett., 8 (2014), 1063-1076. 1

[16] W. Li, X. Wang, N.-J. Huang, Differential inverse variational inequalities in finite dimensional spaces, Acta Math. Sci. Ser. B Engl. Ed., 35 (2015), 407-422. 1, 3.3

[17] X. Li, Y.-Z. Zou, Existence result and error bounds for a new class of inverse mixed quasi-variational inequalities, J. Inequal. Appl., 2016 (2016), 13 pages. 1, 2.4, 3

[18] Z.-H. Liu, S.-D. Zeng, D. Motreanu, Evolutionary problems driven by variational inequalities, J. Differential Equations, 260 (2016), 6787-6799. 1

[19] J.-S. Pang, M. Fukushima, Quasi-variational inequalities, generalized Nash equilibria, and multi-leader-follower games, Comput. Manag. Sci., 2 (2005), 21-56. 1

[20] J.-S. Pang, D. E. Stewart, Differential variational inequalities, Math. Program., 113 (2008), 345-424. 1, 2.1, 2, 2, 3, 3, 4, $4,4,4,4$

[21] A. U. Raghunathan, J. R. Pérez-Correa, E. Agosin, L. T. Biegler, Parameter estimation in metabolic ux balance models for batch fermentation-formulation and solution using differential variational inequalities, Ann. Oper. Res., 148 (2006), 251-270. 1

[22] L. Scrimali, An inverse variational inequality approach to the evolutionary spatial price equilibrium problem, Optim. Eng., 13 (2012), 375-387. 1

[23] M. Sofonea, Y.-B. Xiao, Fully history-dependent quasivariational inequalities in contact mechanics, Appl. Anal., 95 (2016), 2464-2484. 1

[24] F. Vasca, L. Iannelli, M. K. Camlibel, R. Frasca, A new perspective for modeling power electronics converters: Complementarity framework, IEEE Trans. Power Electron., 24 (2009), 456-468. 1

[25] X. Wang, Y.-W. Qi, C.-Q. Tao, Y.-B. Xiao, A class of delay differential variational inequalities, J. Optim. Theory Appl., 172 (2017), 56-69. 1

[26] Y.-B. Xiao, N.-J. Huang, Sub-super-solution method for a class of higher order evolution hemivariational inequalities, Nonlinear Anal., 71 (2009), 558-570. 1

[27] Y.-B. Xiao, N.-J. Huang, Y. J. Cho, A class of generalized evolution variational inequalities in Banach spaces, Appl. Math. Lett., 25 (2012), 914-920.

[28] Y.-B. Xiao, N.-J. Huang, M.-M. Wong, Well-posedness of hemivariational inequalities and inclusion problems, Taiwanese J. Math., 15 (2011), 1261-1276.

[29] Y.-B. Xiao, X.-M. Yang, N.-J. Huang, Some equivalence results for well-posedness of hemivariational inequalities, J. Global Optim., 61 (2015), 789-802. 1

[30] Y. D. Xu, Nonlinear separation approach to inverse variational inequalities, Optimization, 65 (2016), 1315-1335. 1

[31] J.-F. Yang, Dynamic power price problem: an inverse variational inequality approach, J. Ind. Manag. Optim., 4 (2008), 673-684. 1

[32] Y.-H. Yao, M. Postolache, Y.-C. Liou, Z.-S. Yao, Construction algorithms for a class of monotone variational inequalities, Optim. Lett., 10 (2016), 1519-1528. 1

[33] H. Zegeye, N. Shahzad, Y.-H. Yao, Minimum-norm solution of variational inequality and fixed point problem in Banach spaces, Optimization, 64 (2015), 453-471. 1

[34] E. Zeidler, Nonlinear functional analysis and its applications, Translated from the German by the author and Leo F. Boron, Springer-Verlag, New York, (1990). 2.3 